\title{
CARACTERÍSTICAS CLÍNICAS Y PATOLÓGICAS DEL FETO Y DE LA MADRE EN EL ÓBITO FETAL DEL INSTITUTO NACIONAL MATERNO PERINATAL. 2011 AL 2015. LIMA - PERÚ
}

\author{
Jaime Ingar Pinedo', Isabel Advincula Rebbata ${ }^{2}$, Oscar Antonio Limay Rios ${ }^{3}$, Erasmo Huertas Tachinno ${ }^{4}$, \\ Walter Castillo Urquiaga ${ }^{4}$, Walter Ventura Laveriano ${ }^{4}$, Mario Zarate Girao ${ }^{4}$, Antonio Luna Figueroa ${ }^{5}$
}

\begin{abstract}
RESUMEN
Objetivo. Determinar las características clínicas y patológicas del feto y de la madre en el óbito fetal del Instituto Nacional Materno Perinatal del 2011 al 2015. Metodología. Es un estudio de investigación cuantitativo, retrospectivo, observacional, descriptivo y Transversal. Nuestra población es 954 fetos que fallecieron entre la semana 22 de gestación y/o peso mayor de 500 gr en el momento del nacimiento en el Instituto Nacional Materno Perinatal del 2011 al 2015. Donde nuestra muestra es 273 y se aplica el muestreo probabilístico aleatorio simple. La técnica de recolección de datos que se realiza es la observación y el instrumento son los registros donde se encuentran los datos (base de datos e historia clínica) que tiene el Instituto Nacional Materno Perinatal. La técnica para procesar los datos se utilizará el programa SPSS Statístics Base v22.0. En el análisis de datos se estimará por distribución de frecuencias las variables cualitativa, para las variables cuantitativas se estimará medidas de tendencia central (media, mediana) y medidas de dispersión (desviación estándar). Resultados: Una muestra de $273,58.6 \%$ son adultos jóvenes, $52.7 \%$ están cohabitando, $94.5 \%$ son feto único, $67.8 \%$ son multigesta, $94.5 \%$ no tienen antecedentes de aborto, $50.7 \%$ no tienen antecedentes de muerte Fetal. El 81,7\% tiene atención prenatal <6, el 43,2\% tiene sobrepeso, el 14,7\% tiene manifestaciones de preeclampsia, $13,6 \%$ tiene preeclampsia, $91,6 \%$ no tiene enfermedad contagiosa, $56 \%$ son hombres, movimientos fetales están ausentes en $75,1 \%$, el $17,9 \%$, El $62,3 \%$ son cefálicos, el $75,5 \%$ pesan entre 500 y 2500 gramos, el $58,6 \%$ entre 29 y 42 semanas, el $69,2 \%$ son oligohidramnios, el $82,4 \%$ son líquido amniótico, el $38,8 \%$ con anomalías placentarias, el $74,7 \%$ con corioamnionitis y el $70,7 \%$ Ninguna. Conclusión. Al identificar todas las características clínicas y patológicas de la madre concluimos: Las madres: adulto joven, con un estado nutricional: sobrepeso, con estado civil: conviviente, con feto: único, una gravidez: multigesta, en el control prenatal: $<6$, sin antecedentes de óbito fetal ni aborto, cuya característica clínica más frecuente: las manifestación de la preeclampsia, donde la patología es la preeclampsia la más frecuente en el óbito fetal. Al indicar las características clínicas y patológicas del feto concluimos lo más frecuente fueron: El sexo masculino, con presentación: cefálico, cuyo peso oscila entre 500 a 2500 gramos, con una edad gestacional de 29 a 42 semanas, donde los movimientos fetales son ausente y con una restricción del crecimiento intrauterino. Al identificar las características patológicas de la placenta concluimos que lo más frecuente fueron: Oligohidramnios, color verdoso ó meconial, también área de infarto antiguo y reciente: con foco de calcificación e igual para los depósitos de fibrina intervellosa, corioamnionitis y no hubo alteración de cordón.
\end{abstract}

Palabra Clave: Características; Clínico; Patología; Placenta; Óbito Fetal. (Fuente DeCS BIREME).

\section{CLINICAL AND PATHOLOGICAL CHARACTERISTICS OF THE FETUS AND OF THE MOTHER IN THE FETAL DEATH AT THE MATERNAL PERINATAL NATIONAL INSTITUTE. 2011 TO 2015. LIMA - PERU}

\begin{abstract}
Objective. To determine the clinical and pathological characteristics of the fetus and from the mother in the fetal death of the National Maternal Perinatal Institute from 2011 to 2015. Methodology. This is a quantitative, retrospective, observational, descriptive and crosssectional study. Our population is 954 fetuses that died between week 22 of gestation and / or weight greater than $500 \mathrm{gr}$ at the time of birth at the National Maternal Perinatal Institute from 2011 to 2015. Where our sample is 273 and we apply simple random probabilistic sampling. The technique of data collection that is performed is the observation and the instrument are the records where the data (database and medical history) that has the National Maternal Perinatal Institute are found. The technique for processing the data will be the SPSS Statistical Base v22.0 program. In the analysis of data will be estimated by frequency distribution qualitative variables, for quantitative variables will be measured measures of central tendency (mean, median) and measures of dispersion (standard deviation). Results. A sample of $273,58.6 \%$ are young adults, $52.7 \%$ are cohabiting, $94.5 \%$ are single fetus, $67.8 \%$ are multigesta, $94.5 \%$ do not have a history of abortion, $50.7 \%$ do not have a history of death Fetal, $81.7 \%$ have prenatal care $<6,43.2 \%$ are overweight, $14.7 \%$ have manifestations of preeclampsia, $13.6 \%$ have preeclampsia, $91.6 \%$ do not have a communicable disease, $56 \%$ are male, $75.1 \%$ Fetal movements are absent, $17.9 \%$ with IUGR, $62.3 \%$ are cephalic, $75.5 \%$ weigh between 500 and 2500 grams, $58.6 \%$ between 29 and 42 weeks, $69.2 \%$ are oligohydramnios, $82.4 \%$ are amniotic fluid Was greenish, $38.8 \%$ had placental abnormalities, $74.7 \%$ had chorioamnionitis, $70.7 \%$ had no cord alteration. Conclusion. In identifying all the clinical and pathological characteristics of the mother we conclude: Mothers: young adult, with nutritional status: overweight, with marital status: cohabiting, with fetus: single, one pregnancy: multigesta, 6 , with no history of fetal death or abortion, whose clinical characteristic: manifestation of preeclampsia, where the pathology of preeclampsia is the most frequent in fetal death. When indicating the clinical and pathological characteristics of the fetus we conclude the most frequent were: The male sex, with presentation: cephalic, whose weight ranges from 500 to 2500 grams, with a gestational age of 29 to 42 weeks, where fetal movements are absent and With intrauterine growth restriction. When identifying the placental characteristics of the placenta we conclude that the most frequent were: Oligohydramnios, green or meconial color, also area of old and recent infarction: with focus of calcification and equal for intervellose fibrin deposits, chorioamnionitis and no cord alteration.
\end{abstract}

Key Words: Characteristics: Clinical: Pathology: Placenta; Fetal Death. (Source: MeSH NLM).

Médico Gineco-Obstetra Jefe del Servicio de Medicina Fetal del Instituto Nacional Materno Perinatal. Lima-Perú.

Estudiante de Facultad de Medicina Humana de la Universidad San Juan Bautista.Lima-Perú.

Médico Gineco-Obstetra Asistente del Servicio de Medicina Fetal. Jefe de Unidad de Investigación del Instituto Nacional Materno Perinatal. Lima-Perú Médico Gineco-Obstetra Asistente del Servicio de Medicina Fetal del Instituto Nacional Materno Perinatal. Lima-Perú

Médico GínecoObstetra. Profesor Principal de la Universidad Nacional Mayor de San Marcos. Director Ejecutivo de Investigación, Docencia y Atención en Obstetricia y Ginecología del Instituto Nacional Materno Perinatal. Lima-Perú

Recibido: 30-12-16 Aprobado: 30-04-17 


\section{INTRODUCCIÓN}

El Óbito Fetal es un problema que desafortunadamente se presenta con relativa frecuencia originando consecuencias de mucha magnitud para la paciente y también para el médico tratante, el cual casi en un $50 \%$ de los casos no puede darle a la paciente una explicación satisfactoria, dado que suele ser de presentación repentina y en la mayoría de las veces sin ningún síntoma o signo premonitorio.

La muerte fetal, según la Organización Mundial de la Salud, es la pérdida de una gestación in útero a partir de las 22 semanas y un peso mayor a $500 \mathrm{~g}$ en el momento del nacimiento.

El American College of Obstetrician and Gynecologists la define como la pérdida de una gestación in utero de 20 semanas de gestación y mayor a $500 \mathrm{~g}$ en el momento del nacimiento.

La muerte fetal es una catástrofe obstétrica además de ser un duro golpe para las familias y la madre en quienes crea un sentimiento de culpa y miedo a salir nuevamente embarazada por el temor a que este problema se repita. Reportes en la literatura demuestran que sólo en el $50 \%$ a $70 \%$ de los casos de muerte fetal se determina una causa específica.

El estudio de las causas a través del diagnóstico anatomopatológico y la identificación de los factores maternos que se asocian a esta entidad cobran importancia en el contexto de buscar factores en los que se pueda trabajar para evitarlos.

En las bibliografía mundial se han reportado varios factores socio-demográficos, obstétricos y médicos asociados a muerte fetal siendo los más importantes no controles prenatales, edad materna > 35 años; retardo crecimiento intrauterino $(\mathrm{RCIU})$, paridad $>4$, abortos previos, antecedente de muerte fetal, período intergenésico < a 6 meses, hemorragia del tercer trimestre (HTT), preeclampsia, eclampsia, malformaciones fetales.

\section{MATERIALES Y MÉTODOS}

El presente estudio es una investigación cuantitativo. Retrospectivo, Observacional, Descriptivo, Transversal. La población de estudio está conformada por un total de 954 fetos que fallecieron entre la semana 22 de gestación y/o peso mayor de $500 \mathrm{gr}$ en el momento del nacimiento en el Instituto Nacional Materno Perinatal del 2011 al 2015 .

La muestra es de 273 óbitos fetales y el muestreo es probabilístico aleatorio simple. La técnica de recolección de datos que se realiza en este proyecto la observación y el instrumento son los registros donde se encuentran los datos (base de datos e historia clínica) que tiene el Instituto Nacional Materno Perinatal. Se utiliza el procesamiento de datos del programa SPSS Statístics Base v.22.0. Para el análisis de datos se empieza con el traslado de la información a la Ficha técnica de recolección de datos.

Se ordenan las fichas por año de defunción de los óbitos fetales y se analizan de acuerdo a la frecuencia y a las tablas de contingencia las variables independientes, dependiente e interviniente. Los datos son tabulados por el programa Microsoft Excel y procesado por el SPSS versión 22.

\section{RESULTADOS}

Se tomó una muestra de 273 óbitos fetales siendo 2011 $=48 ; 2012=46 ; 2013=57 ; \quad 2014=57 ; 2015=$ 65. En las edades maternas: Adolescentes 17,6\%, adulto joven $58,6 \%$ y adulto añosa $23,8 \%$. Características clínicas de la madre: manifestación preeclampsia 14,7\%, pérdida de líquido amniótico $12,5 \%$, sangrado vaginal $9,9 \%$, dolor hipogástrico con síntomas de infección de tracto urinario (ITU) 9,9\%, elevación presión arterial 7,7\%, dolor hipogástrio con sangrado vaginal $7,7 \%$, signo y síntomas de ITU 6,20\%, dolor en hipogastrio 5,5\%. En enfermedades no transmisible de la madre: preeclampsia $13,6 \%$, ITU con anemia $12,8 \%$, ruptura prematura de membranas con anemia 10,3\%, hipertension gestacional $8,4 \%$. Enfermedades transmisible de la madre; ninguno $91,6 \%$, sífilis $1,8 \%$, tuberculosis pulmonar $1,5 \%$, hepatitis $1,5 \%$. Estado civil de la madre: soltera $15 \%$, casada $32,2 \%$, conviviente $52,7 \%$. Cantidad de feto en el embarazo: único $94,5 \%$ mellizos $5,5 \%$, gravidez de la madre: primigesta $29,3 \%$, multigesta $67,8 \%$, gran multigesta $2,9 \%$. Antecedente de óbito fetal $5,5 \%$ y no antecedente $94,5 \%$. Antecedentes de aborto 40,3\%. Sin antecedente $50,7 \%$. Control prenatal $<6 \quad 81,7 \%$ y $\geq 618,3 \%$, Estado nutricional de la madre sobrepeso $43,2 \%$, normal $32,2 \%$, obesidad I $17,9 \%$. Características clínicas del feto: movimiento fetal ausente $75,1 \%$, latidos cardiacos ausente 19\%, confirmación ecográfico obstétrico del óbito fetal RCIU 17,9\%, hipoxia fetal 15\%, Hematoma retroplacentario y subamniótico $11,7 \%$, hidrops fetal $11,4 \%$, vejez prematura placentaria $8,4 \%$, higroma quístico 5,9\%. Presentación del óbito fetal cefálico $62,3 \%$, podálico $32,6 \%$, transverso $2,9 \%$. Peso al momento del parto: $500-2500 \mathrm{~g} 75,5 \%$; 2501 - 4500 g 23,1\%; $4501-6500$ g 1,5\%. Edad gestacional 22-28 Semanas $41,4 \%$, $29-42$ semanas $58,6 \%$. Sexo del feto en el óbito fetal masculino $56 \%$, femenino $43,6 \%$, indefinido $0,4 \%$, color del líquido amniótico acrómico $6,2 \%$, meconial $82,4 \%$ amarillo $0,4 \%$, rojo marrón $11 \%$. Anomalías placentarias:area de infarto antiguo y reciente con foco de calcificación $38,8 \%$, área de infarto antiguo y reciente depósito de fibrina intervellosa $38,8 \%$, aumento leve de nudo sinciciales y depósitos fibrinoides intervellosos $8,8 \%$; area de infarto antiguo y reciente $7,7 \%$, hemorragia 
intervellosa deciduitis 2,6\%, sin alteración 1,8\%; focos escasos de calcificación 1,5\%. Anomalias de membrana: sin alteración $7 \%$, corioamnionitis $74,7 \%$, macrófago cargado de meconio 18,3\%. Anomalías de cordón: sin alteración 70,70\%, edema vellositario $29,3 \%$. Alteración del líquido amniótico: anhidramnio 2,2\%, oligohidramnio $69,2 \%$, polihidramnios $28,6 \%$.

Tabla 1. Frecuencias de madres en las que se produce óbito fetal.

\begin{tabular}{lcc}
\hline \multicolumn{1}{c}{ Edad Materna } & Frecuencia & Porcentaje \\
\hline Adolescentes & 48 & $17,60 \%$ \\
\hline Adulto Joven & 160 & $58,60 \%$ \\
Adulto Añosa & 65 & $23,80 \%$ \\
Total & 273 & $100 \%$ \\
\hline
\end{tabular}

Fuente: Historias Clínicas con óbitos fetales del Instituto Nacional Materno Perinatal. 2011- 2015

Tabla 2. Características clínicas de la madre con óbito fetal.

\begin{tabular}{|c|c|c|}
\hline Características Clínicas De La Madre & Frecuencia & $\%$ \\
\hline Manifestación Preeclampsia & 40 & $14.70 \%$ \\
\hline Pérdida de Líquido Amniótico & 34 & $12.50 \%$ \\
\hline Sangrado Vaginal & 27 & $9.90 \%$ \\
\hline $\begin{array}{l}\text { Dolor Hipogastrio _ Signo y Síntoma } \\
\text { Del Itu }\end{array}$ & 27 & $9.90 \%$ \\
\hline Elevación Presión Arterial & 21 & $7.70 \%$ \\
\hline Dolor Hipogastrio_Sangrado Vaginal & 21 & $7.70 \%$ \\
\hline Signo y Síntoma Del Itu & 17 & $6.20 \%$ \\
\hline Dolor en Hipogastrio & 15 & $5.50 \%$ \\
\hline $\begin{array}{l}\text { Dolor Hipogastrio_Manif. } \\
\text { Preeclampsia_Sang. Vaginal }\end{array}$ & 12 & $4.40 \%$ \\
\hline $\begin{array}{l}\text { Pérdida L.A._Sang. Vaginal_Manif. } \\
\text { Preeclampsia }\end{array}$ & 11 & $4.00 \%$ \\
\hline $\begin{array}{l}\text { Pérdida L.A_Elevación De Alza } \\
\text { Térmica }\end{array}$ & 11 & $4.00 \%$ \\
\hline $\begin{array}{l}\text { Signo y Síntomas DM_Manifestación } \\
\text { Preeclampsia }\end{array}$ & 10 & $3.70 \%$ \\
\hline Asintomático & 7 & $2.60 \%$ \\
\hline Crisis de Epilepsia & 6 & $2.20 \%$ \\
\hline $\begin{array}{l}\text { Manifestación Enfermedad } \\
\text { Respiratorio }\end{array}$ & 4 & $1.50 \%$ \\
\hline Manifestaciones De La Sífilis & 3 & $1.10 \%$ \\
\hline Contracciones Uterinas & 3 & $1.10 \%$ \\
\hline Vómito Líquido Amarillo & 1 & $0.40 \%$ \\
\hline Convulsiones Tónico Clónicas & 1 & $0.40 \%$ \\
\hline $\begin{array}{l}\text { Síndrome Crest_Pérdida Líquido } \\
\text { Amniótico }\end{array}$ & 1 & $0.40 \%$ \\
\hline $\begin{array}{l}\text { Manifestación Neumonía } \\
\text { Manifestación Dpp }\end{array}$ & 1 & $0.40 \%$ \\
\hline Total & 273 & $100 \%$ \\
\hline
\end{tabular}

Fuente: Historias Clínicas con óbitos fetales del Instituto Nacional Materno Perinatal. 2011- 2015
Tabla 3. Características patológicas: Enfermedades no transmisible de la madre con óbito fetal.

\begin{tabular}{lcc}
\hline Enfermedad No Transmisible & Frecuencia & Porcentaje \\
\hline Preeclampsia & 37 & $13.60 \%$ \\
\hline ITU_Anémia & 35 & $12.8 \%$ \\
\hline Anémia_RPM & 28 & $10.3 \%$ \\
\hline HTGestacional & 23 & $8.4 \%$ \\
\hline Ninguno & 20 & $7.30 \%$ \\
\hline Hemorrágia & 17 & $6.2 \%$ \\
\hline RPM_Enf. Cardiovascular & 12 & $4.40 \%$ \\
\hline RPM_Preeclamp_Anémia & 12 & $4.4 \%$ \\
\hline DPP_Anémia & 12 & $4.4 \%$ \\
\hline ITU & 12 & $4.4 \%$ \\
\hline DPP_PreEclamp._Anémia & 11 & $4.00 \%$ \\
\hline DM y Preeclampsia & 10 & $3.7 \%$ \\
\hline RPM_PreEclamp_HTG & 7 & $2.60 \%$ \\
\hline Epilepsia & 7 & $2.60 \%$ \\
\hline RPM_Enf. Renal & 7 & $2.6 \%$ \\
\hline DPP_ITU & 4 & $1.5 \%$ \\
\hline Enfermedad respiratoria & 4 & $1.5 \%$ \\
\hline Preeclamp_HTA_Anemia & 3 & $1.10 \%$ \\
\hline Enfermedad renal_HTA & 2 & $0.7 \%$ \\
\hline RPM_Sepsis_mioma uterino & 2 & $0.7 \%$ \\
\hline Preeclamp_Disf. Hepat_Renal_ & 1 & $0.40 \%$ \\
Anémia & 1 & $0.4 \%$ \\
\hline Eclampsia & 1 & $0.4 \%$ \\
\hline Preeclamp_ITU & 1 & $0.4 \%$ \\
\hline Esclerodermia_RPM & 1 & $0.4 \%$ \\
\hline DPP_disf. Renal_anémia & 1 & $0.4 \%$ \\
\hline Incompetencia cervical & 1 & $0.4 \%$ \\
\hline Rotrura Precoz Membrana & $0.4 \%$ \\
\hline DPP_Miomatosis & $100 \%$ \\
\hline Total & & \\
\hline & & \\
\hline & 17 & \\
\hline
\end{tabular}

Fuente: Historias Clínicas con óbitos fetales del Instituto Nacional Materno Perinatal. 2011- 2015.

Tabla 4. Características patológicas: Enfermedades transmisibles de las madres con óbito fetal.

\begin{tabular}{lcc}
\hline \multicolumn{1}{c}{ Enfermedad Transmisible } & Frecuencia & Porcentaje \\
\hline Ninguno & 250 & $91.60 \%$ \\
\hline Sífilis & 5 & $1.80 \%$ \\
\hline TBC & 4 & $1.50 \%$ \\
\hline Hepatitis & 4 & $1.50 \%$ \\
\hline Sífilis_TBC & 2 & $0.70 \%$ \\
Rubeola_citomegalovirus & 2 & $0.70 \%$ \\
VIH & 2 & $0.70 \%$ \\
Neumonía & 1 & $0.40 \%$ \\
VPH & 1 & $36.60 \%$ \\
tifoidea & 1 & $0.40 \%$ \\
Fiebre amarilla & 1 & $0.40 \%$ \\
\hline \multicolumn{1}{c}{ Total } & 273 & $100 \%$ \\
\hline
\end{tabular}


Tabla 5. Características clínicas del feto con óbito fetal.

\begin{tabular}{lcc}
\hline Características clínicas óbito fetal & Frecuencia & Porcentaje \\
\hline Movimiento fetal ausente & 205 & $75.10 \%$ \\
\hline Latidos cardiacos Ausente & 52 & $19.00 \%$ \\
Movimiento fetal disminuido & 15 & $5.50 \%$ \\
\hline Latidos cardiacos disminuido & 1 & $0.40 \%$ \\
\hline Total & 273 & $100 \%$ \\
\hline
\end{tabular}

Fuente: Historias Clínicas con óbitos fetales del Instituto Nacional Materno Perinatal. 2011- 2015

Tabla 6. Características patológicas: Informe ecográfico obstétrico del óbito fetal.

\begin{tabular}{lcc}
\hline Informe Ecográfico Obstétrico & Frecuencia & Porcentaje \\
\hline RCIU & 49 & $17.90 \%$ \\
Hipoxia fetal & 41 & $15.00 \%$ \\
\hline Hematoma retroplacentario y & 32 & $11.70 \%$ \\
subamniótico & 31 & $11.40 \%$ \\
\hline Hidrops fetal & 23 & $8.40 \%$ \\
\hline Vejez_prematura_placentaria & 16 & $5.90 \%$ \\
Higroma quístico & 13 & $4.80 \%$ \\
\hline Higroma quistica_Hidrops fetal & 10 & $3.70 \%$ \\
\hline Sin alteracion & 9 & $3.30 \%$ \\
Circular al cuello & 8 & $2.90 \%$ \\
\hline Causa no específica & 8 & $2.90 \%$ \\
Malformación genética & 8 & $2.90 \%$ \\
\hline Malformación cromosómica & 7 & $2.60 \%$ \\
\hline Síndrome de transfusión feto_fetal & 7 & $2.60 \%$ \\
\hline Signos de desprendimiento & 5 & $1.80 \%$ \\
prematuro de placenta & 3 & $1.10 \%$ \\
\hline Anoxia intrauterino & 2 & $0.70 \%$ \\
\hline Anancefalia_acrania & 1 & $0.40 \%$ \\
\hline macrosomia & 273 & $100 \%$ \\
\hline Anancefálico_Sindrome de & 5 transfusión feto_fetal & \\
\hline Total & &
\end{tabular}

Fuente: Historias Clínicas con óbitos fetales del Instituto Nacional Materno Perinatal. 2011- 2015

Tabla 7. Frecuencia del peso en el momento del parto.

\begin{tabular}{ccc}
\hline Peso Del Óbito Fetal & Frecuencia & Porcentaje \\
\hline $500-2500 \mathrm{gr}$ & 206 & $75.50 \%$ \\
$2501-4500 \mathrm{gr}$ & 63 & $23.10 \%$ \\
$4501-6500 \mathrm{gr}$ & 4 & $1.50 \%$ \\
TOTAL & 273 & $100 \%$ \\
\hline
\end{tabular}

Fuente: Historias Clínicas con óbitos fetales del Instituto Nacional Materno Perinatal. 2011- 2015

Tabla 8. Frecuencia en la edad gestacional.

\begin{tabular}{ccc}
\hline Edad Gestacional & Frecuencia & Porcentaje \\
\hline 22-28 Semanas & 113 & $41.40 \%$ \\
29-42 Semanas & 160 & $58.60 \%$ \\
Total & 273 & $100 \%$ \\
\hline
\end{tabular}

Fuente: Historias Clínicas con óbitos fetales del Instituto Nacional
Materno Perinatal. 2011- 2015

Tabla 9. Frecuencia en las anomalías placentarias en los óbitos fetales.

\begin{tabular}{lcc}
\hline \multicolumn{1}{c}{ Anomalías Placentarias } & Frecuencia & Porcentaje \\
\hline $\begin{array}{l}\text { Área de Infarto Antiguo y Reciente } \\
\text { con foco de calcificación }\end{array}$ & 106 & $38.80 \%$ \\
$\begin{array}{l}\text { Área de infarto Antiguo y Reciente } \\
\text { Depósito de fibrina intervellosa }\end{array}$ & 106 & $38.80 \%$ \\
$\begin{array}{l}\text { Aumento leve de nudo sinciciales y } \\
\text { depósitos fibrinoides intervellosos }\end{array}$ & 24 & $8.80 \%$ \\
\hline $\begin{array}{l}\text { Area de Infarto Antiguo y Reciente } \\
\text { Hemorragia Intervellosa_Deciduitis }\end{array}$ & 21 & $7.70 \%$ \\
\hline Sin alteración & 7 & $2.60 \%$ \\
\hline Focos Escasos de Calcificación & 5 & $1.80 \%$ \\
\hline
\end{tabular}

Fuente: Historias Clínicas con óbitos fetales del Instituto Nacional Materno Perinatal. 2011- 2015

Tabla 10. Frecuencia en las Anomalías de Membrana en los Óbitos Fetales.

\begin{tabular}{|c|c|c|}
\hline Anomalias De La Membrana & Frecuencia & Porcentaje \\
\hline Sin Alteración & 19 & $7.00 \%$ \\
\hline Corioamnionitis & 204 & $74.70 \%$ \\
\hline Macrófago Cargado De Meconio & 50 & $18.30 \%$ \\
\hline Total & 273 & $100 \%$ \\
\hline \multicolumn{3}{|c|}{$\begin{array}{l}\text { Fuente: Historias Clínicas con óbitos fetales del Institutc } \\
\text { Nacional Materno Perinatal. 2011- } 2015\end{array}$} \\
\hline \multicolumn{3}{|c|}{$\begin{array}{l}\text { Tabla 11. Frecuencia en anomalías de cordón en los } \\
\text { óbitos fetales. }\end{array}$} \\
\hline Anomalias De Cordón & Frecuencia & Porcentaje \\
\hline Sin Alteración & 193 & $70.70 \%$ \\
\hline Edema Vellositario & 80 & $29.30 \%$ \\
\hline Total & 273 & $100 \%$ \\
\hline
\end{tabular}

Fuente: Historias Clínicas con óbitos fetales del Instituto Nacional Materno Perinatal. 2011- 2015

Tabla 12. Frecuencia en alteración de la cantidad del líquido amniótico.

\begin{tabular}{lcc}
\hline $\begin{array}{c}\text { Alteración Del Liquido } \\
\text { Amnniotico }\end{array}$ & Frecuencia & Porcentaje \\
\hline Anhidramnio & 6 & $2.20 \%$ \\
Oligohidramnios & 189 & $69.20 \%$ \\
Polihidramnios & 78 & $28.60 \%$ \\
Total & 273 & $100 \%$ \\
\hline
\end{tabular}

Fuente: Historias Clínicas con óbitos fetales del Instituto Nacional Materno Perinatal. 2011- 2015

\section{DISCUSIÓN}

La edad materna en el estudio se presentó con mayor frecuencia ( 160 casos) en óbitos fetales con un porcentaje de $58,60 \%$ en los adultos jóvenes (20 - 34 años de edad), donde Milla $L$ y Colaboradores en su estudio muerte fetal en el Hospital Nacional Arzobispo Loayza-2003, 
que la edad menor de 35 años hubo mayor porcentaje de $76,59 \%$ siendo similar al presente estudio; en cambio, difiere al estudio de Moreno $\mathrm{N}$ en factores que se asocian a la muerte fetal intrauterina en el Hospital María Auxiliadora-2011, la edad con mayor porcentaje fue en el grupo etareo entre 36 a 45 años con un $38,30 \%$.

Con respecto a las características clínicas y patológicas de las madres observamos en el estudio la presencia de mayor frecuencia (37 casos) para la preeclampsia con un $14,7 \%$, donde la similitud lo tiene el estudio de Milla L y Colaboradores en muerte fetal en el Hospital Nacional Arzobispo Loayza-2003, donde la preeclampsia es de un $8,69 \%$, por el contrario el estudio de Moreno $\mathrm{N}$ en Factores que se asocian a la muerte fetal intrauterina en el Hospital María Auxiliadora-2011, coloca a la anémia con mayor porcentaje de $66 \%$.

El estado civil de las madres en el estudio representan con mayor frecuencia (144 caos) a las convivientes con un $52,70 \%$ que tuvieron óbitos fetales, de igual forma el estudio de Moreno $\mathrm{N}$ en factores que se asocian a la muerte fetal intrauterina en el Hospital María Auxiliadora-2011, de un 72,3\% y diferente al estudio de Huiza L y Colaboradores en la muerte fetal y la muerte neonatal tiene origen multifactorial de 1996 al 2000, donde más frecuente es el estado civil soltera con un $14 \%$.

La gravidez en el estudio representa con mayor frecuencia (185 caos) a las multigestas con un $67,8 \%$, de igual forma en el estudio de Milla $L$ y Colaboradores en muerte fetal en el Hospital Nacional Arzobispo Loayza-2003, las multigestas tiene un $86,96 \%$, asi de igual manera los estudios de Moreno $\mathrm{N}$ en Factores que se asocian a la muerte fetal intrauterina en el Hospital María Auxiliadora-2011, con un 46,2\%.

En el estudio no fue frecuente los antecedentes de aborto de igual manera para el estudio de Moreno $\mathrm{N}$ en Factores que se asocian a la muerte fetal intrauterina en el Hospital María Auxiliadora-2011, pero si hubo antecedentes en el estudio de Huiza $L$ y Colaboradores en la Muerte fetal y la muerte neonatal tiene origen multifactorial de 1996 al 2000 , con un $23,5 \%$.

En el presente estudio el sexo más frecuente en los óbitos fetales es masculino (153 casos) teniendo un $56 \%$ de igual forma para el estudio de Huiza $L$ y Colaboradores en la Muerte fetal y la muerte neonatal tiene origen multifactorial de 1996 al 2000, y del estudio de Moreno N en Factores que se asocian a la muerte fetal intrauterina en el Hospital María Auxiliadora-2011.

La posición más frecuente en el estudio fue cefálico (170 casos) de un $62,30 \%$ no así en el estudio de Huiza $L$ y Colaboradores en la muerte fetal y la muerte neonatal tiene origen multifactorial de 1996 al 2000, que fue podálico en un $16,2 \%$.

El peso de los óbitos fetales en el estudio entre los 500 a
2500 gramos con una frecuencia de 206 y un porcentaje de $75,5 \%$ donde el estudio de Milla $L$ y Colaboradores en muerte fetal en el Hospital Nacional Arzobispo Loayza-2003, se aproxima a la similitud donde el peso es de 500 a 3950 gramos de igual manera para el estudio de Huiza $L$ y Colaboradores en la muerte fetal y la muerte neonatal tiene origen multifactorial de 1996 al 2000 , cuyo peso son menores de 2500 con un 62,5\%.

La edad gestacional en el estudio con más frecuencia fue de 160 óbitos fetales de un 58,6\% entre las 29 42 semanas, donde el estudio Milla $L$ y Colaboradores en Muerte fetal en el Hospital Nacional Arzobispo Loayza-2003, cursan entre 20 a 40 semanas.

En el estudio los controles prenatales tienen más frecuencia (223 casos) los menores de 6 atenciones con un $81,7 \%$, donde no hay similitud con el estudio de Moreno $\mathrm{N}$ en Factores que se asocian a la muerte fetal intrauterina en el Hospital María Auxiliadora-2011, donde el control prenatal fue completo mayor o igual de 6 siendo un $79,2 \%$.

En el estudio se observa que hubo anomalías placentarias en un $38,8 \%$ donde hubo similitud en el estudio de Milla $L$ y Colaboradores en Muerte fetal en el Hospital Nacional Arzobispo Loayza-2003, siendo un 23,29\%.

El líquido amniótico del estudio se observa más frecuente (189 casos) a los oligohidramnios con un $69,2 \%$, no así fue le estudio de Moreno $\mathrm{N}$ en Factores que se asocian a la muerte fetal intrauterina en el Hospital María Auxiliadora-2011, que el líquido amniótico fue normal en un $77,7 \%$.

En la anomalías del cordón el estudio no hubo alteración en un $70,70 \%$ así mismo para el estudio de Moreno $\mathrm{N}$ en Factores que se asocian a la muerte fetal intrauterina en el Hospital María Auxiliadora-2011, con un 86,2\%.

En el estudio el estado nutricional de la madre son más frecuente (118 casos) el sobrepeso con un 43,20\% a diferencia del estudio de Huiza $L$ y Colaboradores en la muerte fetal y la muerte neonatal tiene origen multifactorial de 1996 al 2000, donde el más frecuente fue la obesidad en un $30,80 \%$.

\section{CONCLUSIONES}

Al identificar todas las características clínicas de la madre, lo más frecuente son las manifestaciones de la preeclampsia y en las características patológicas de la madre, lo más frecuente son la Preeclampsia como una enfermedad no transmisible y como la enfermedad transmisible la sífilis.

Teniendo otras características como las madres adultas joven, con un estado nutricional de sobrepeso, con estado civil: conviviente, de feto único, multigesta, control 
prenatal $<6$, sin antecedentes de óbito fetal ni aborto. Al indicar las características clínicas del feto, lo más frecuente son la ausencia de movimientos fetales y en las características patológicas del feto es la restricción de crecimiento intrauterino (RCIU).

Teniendo otras características como de sexo masculino, presentación cefálico, peso entre 500 a 2500 gramos, edad gestacional de 29 a 42 semanas.

Al identificar las características patológicas de la placenta concluimos lo más frecuente son: oligohidramnios, líquido meconial, área de infarto antiguo y reciente: con focos de calcificación e igual para los depósitos de fibrina intervellosa, corioamnionitis y no hubo alteración de cordón.

\section{REFERENCIAS BIBLIOGRAFICAS}

1. Linares J, Poulsen R. Muerte fetal in útero: Etiología y factores asociados en un Hospital Regional de Antofagasta, Chile. CIMEL 2007; 12(1):1-11.

2. Instituto Nacional Estadística e Informática. Encuesta Demográfica y de Salud Familiar- ENDES 2014. Lima - Perú. 2015:1-490.

3. Milla L, Saravia N, Salviz M, y Rojas J. Muerte Fetal en el Hospital Nacional Arzobispo Loayza durante periodo Agosto 2003 - Noviembre 2004. Rev Med Hered. 2005; 16(4): 260 265.

4. Aragón V. Características Histopatológicas placentarias provenientes de óbito Fetal y valor del examen de la placenta en la autopsia fetal. Optar Título de Especialidad en Anatomía Patológica. 2007. Lima - Perú.

5. Moreno N. Factores que se asocian a la muerte fetal intrauterina presentados en el Hospital María Auxiliadora del Cono Sur de Lima 2011. Rev Perú Obstet Enferm. 2012;8(2):56-66.Lima - Perú.

6. Huiza $L$ y col. La muerte fetal y la muerte neonatal tienen origen multifactorial. Anales 2003;64(1):13 - 20. Lima - Perú.

7. Vera E. Epidemiología de la muerte intrauterina en el Hospital san Bartolomé 2002 (Tesis de especialista en Ginecoobstericia). Lima-Perú: Universidad Nacional Mayor de San Marcos. 2002.

8. Pons A, Sepúlvedas E, Leiva JL, Rencoret G, Germain A. Muerte fetal. Rev Med Clin Condes. 2014; 25(6)908 - 916.

9. Rivas E y Col. Obito Fetal: Hallazgos de patología en una Institución de alta complejidad. Rev Colomb Obstet Ginecol 2012; 63: 376 - 381 .

10. Molina S, Alejandra D. Muerte fetal anteparto: ¿es una condición prevenible?. Univ Med Bogota(Colombia) 2010;51(1):59-73.

11. Trejo K, Ávila J, Pardo R. Índice de muerte fetal tardía y factores de riesgo obstétricos, perinatales y socioeconómicos asociados. Bogotá - Colombia. Arch Inv Mat Inf. 2012;4(2):71 - 78.

12. Fernández $M$, Cruceyra $M$, Rodríguez $R$, Magdaleno $F$, Omeñaca F, González A. Gestación gemelar con muerte intraútero de uno de los fetos: pronóstico materno y neonatal del feto superviviente. España. Arch Inv Mat Inf. 2012;4(2):71-78.

13. Pérez L, Morales N. Óbito Fetal. Tegucigalpa - Honduras.
Rev Med Hondur. 1982; 50:1-3.

14. Nava C. La maternidad en la adolescencia, en contribuciones a las ciencias sociales. Noviembre 2009. Málaga - España. 2009. Disponible en: www.eumed.net/rev/cccss/06/cmnf.htm 18/09/2016.

15. Cortejoso D. Riesgos y consejos para la embarazada añosa (mayor de 35 años). 2010. Disponible en: http://www. cometelasopa.com/riesgos-y-consejos-para-la-embarazada.

16. Gonce A, Boguiña J, Marimon E, Muñoz M, Palacios M, Martinez J, et al. Asistencia al embarazo y parto de gestantes múltiples.2015:1-22.

17. Rencoret G. Embarazo Gemelar. Rev Med Condes. 2014;23(6):964-971.

18. Caballero L, Carvajal V, Segura J, Quintanilla A, Valencia G, García J. Diagnóstico y manejo del embarazo múltiple. Disponible en: www.cenetec.salud.gob.mx/interior/gpc. html

19. Cuidados del cordón umbilical en el recién nacido: revisión de la evidencia científica Umbilical Cord Care Procedures in the Newborn: A Systematic. Disponible en: http://revistaseug.ugr. es/index.php/ars/article/view/4380/4371

20. Kaneshiro N. Clínica Dam en Madrid. Disponible en : https:// www.clinicadam.com/salud/5/002367.html. (consultado el 19/09/2016)

21. Grandi C, López F. Estimación de la edad gestacional: Revisión de la Literatura. Rev del Hospital Materno Infantil Ramón Sardá. Buenos Aires, Argentina. 2004;23(3):138-143.

22. Gil A. Embarazo: diagnóstico, edad gestacional y fecha del parto. Medisan 2008; 12(4).

23. Salen L, Singler K. Rh incompatibility. Emedicine .com 2009.

24. Frenk J, Ruelas E, Tapia R, Castañón R, De León M, González E, et al. Prevención, diagnóstico y manejo de la Preeclampsia / Eclampsia. Salud Contigo es posible. $3^{\text {a }}$ edición junio 2002:43pp.

25. Cararach V, Botet F. Preeclampsia. Eclampsia y Síndrome Hellp. Asociación Española Pediátrica.2008. Disponible en: www.aeped.es/protocolos/

26. SEGO. Protocolos asistenciales de la Sociedad Española de Ginecología y Obstetricia. Rotura prematura de membrana. 2012. Disponible en. http://www.elsevier.es.( Consultado el 28/09/2016)

27. Cruz M, Redondo R, Caño A, Carretero P, Padilla C, Ruiz A. Análisis de la mortalidad perinatal en nacidos con peso menor de 1000 gramos en el Hospital San Cecilio de Granada(España) durante el período 1991 - 2010. Arch Argent Pediatr. 2013;111(1):45-52.

28. Aller J, Pagés G. Obstetricia Moderna. Tercera Edición. Mc Graw - Hill - Interamericana. 1999:1-584.

29. Kaneshiro N. Clínica Dam en Madrid. Disponible en https:// www.clinicadam.com/salud/5/002367.html. Consultado el 19/09/2016.

30. Vigil P, Savransky R, Pérez J, Delgado J, Nuñez E. Ruptura prematura de membrana. Guía clínica de la Federación Latino Americana de Sociedades de Ginecología y Obstetricia. 2011;1:1-19.

31. Torres M. Determinar la frecuencia de las causas predisponentes del óbito fetal en pacientes atendidas en el Hospital Materno Infantil Dra. Matilde Hidalgo de Procel en el lapso (Tesis). Guayaquil - Ecuador. 2013.

32. Caro J, Riaño G. Guía de óbito fetal. Hospital la vistoria. 2011: 2.

33. International Diabetes Federation. Diabetes. Atlas. 2015:7. 